\title{
Strong Transitivity and Graph Maps
}

by

\author{
Katsuya YOKOI
}

Presented by Andrzej LASOTA

Dedicated to Professor Takao Hoshina on his 60th birthday

\begin{abstract}
Summary. We study the relation between transitivity and strong transitivity, introduced by W. Parry, for graph self-maps. We establish that if a graph self-map $f$ is transitive and the set of fixed points of $f^{k}$ is finite for each $k \geq 1$, then $f$ is strongly transitive. As a corollary, if a piecewise monotone graph self-map is transitive, then it is strongly transitive.
\end{abstract}

1. Introduction. The purpose of this paper is to study (strong) transitivity properties for graph self-maps. W. Parry [7] pointed out a sufficient condition for the existence of a special measure on a symbolic dynamics, which has a close relation to a linearization of the dynamics on intervals. Then, as an application, he introduced the concept of strong transitivity that is one of conditions under which an interval map is conjugate to a uniformly piecewise linear map $[7, \S 5, \S 6]$. E. Coven and I. Mulvey [6, Theorem $\mathrm{B}$ and $\mathrm{C}]$ stated the relation between transitivity and strong transitivity properties for interval (or circle) self-maps.

In this paper, we extend the above relation to graph self-maps (see §4). A motivation for studying graph maps is that higher-dimensional dynamics can often be reduced to one-dimensional dynamics: this is the case in the study of the structure of attractors of a diffeomorphism, the quotient maps generated by maps on manifolds with an invariant foliation of codimension one and the dynamics of pseudo-Anosov homeomorphisms on a surface.

2000 Mathematics Subject Classification: 37E25, 37B20.

Key words and phrases: transitive, strongly transitive, graph.

The author was partially supported by the Grant-in-Aid for Scientific Research (C) (No. 16540067), the Ministry of Education, Culture, Sports, Science and Technology of Japan. 
Throughout this paper, by a graph we mean a connected compact onedimensional polyhedron, and a tree is a graph which contains no loops. We also assume that any graph $G$ is endowed with a metric $d$; we define $\mathbb{B}(x ; \varepsilon)$, $x \in G, \varepsilon>0$, to be the set of points of $G$ whose distance from $x$ is less than $\varepsilon . \mathrm{B}(G)$ and $\mathrm{E}(G)$ denote the sets of branch points and of endpoints of $G$, respectively. A map $f$ is a continuous function from a space $X$ to itself; $f^{0}$ is the identity map, and for every $n \geq 0, f^{n+1}=f^{n} \circ f$. We denote by $\operatorname{Fix}(f)$ and $\operatorname{Per}(f)$ the sets of fixed points and of periodic points of $f$, respectively. For a subset $K$ of $X$, Int $K$ and $\mathrm{Cl} K$ denote the interior and closure of $K$ in $X$.

2. Preliminaries. An onto map $f: X \rightarrow X$ is called (topologically) transitive if any of the following equivalent conditions holds.

(1) There exists a point with dense orbit.

(2) Whenever $U, V$ are non-empty open sets, there exists an $n \geq 1$ such that $f^{-n}(U) \cap V \neq \emptyset$.

(3) The only closed invariant set $K$ with $\operatorname{Int} K \neq \emptyset$ is $K=X$.

REMARK. We note that, in the case of a graph map $f: G \rightarrow G, f$ is transitive if and only if for every pair of non-empty open sets $U$ and $V$ in $G$, there exists a $k \geq 1$ such that $U \cap \operatorname{Int} f^{k}(V) \neq \emptyset$.

We first recall some basic, but important results for transitive graph maps.

THEOREM 2.1 ([4]). Let $f: G \rightarrow G$ be a transitive graph map without periodic points. Then $G$ is the circle and $f$ is conjugate to an irrational rotation.

Theorem 2.2 ([4]). Let $f: G \rightarrow G$ be a transitive graph map with periodic points. Then the set of periodic points of $f$ is dense in $G$.

In the study of transitive maps, the subclass of those maps having all iterates transitive plays a significant role. A map $f$ is totally transitive if $f^{n}$ is transitive for all $n \geq 1$ (see [1]); note that a transitive map is not always totally transitive.

The following splitting theorem is quite useful, since it allows us to reduce the study of transitive graph maps to that of totally transitive graph maps.

TheOrem 2.3 ([1], [2], [5]). Let $f: G \rightarrow G$ be a transitive graph map. Then exactly one of the following two statements holds:

(1) $f$ is totally transitive.

(2) There exist a $k>1$ and non-degenerate connected subgraphs $G_{0}, \ldots$ $\ldots, G_{k-1}$ of $G$ such that

(a) $G=\bigcup_{i=0}^{k-1} G_{i}$ 
(b) $G_{i} \cap G_{j}=\mathrm{E}\left(G_{i}\right) \cap \mathrm{E}\left(G_{j}\right)$ for $i \neq j$,

(c) $f\left(G_{i}\right)=G_{i+1(\bmod k)}$ for $i=0, \ldots, k-1$,

(d) $\left.f^{k}\right|_{G_{i}}$ is totally transitive for $i=0, \ldots, k-1$.

3. Strong transitivity. A map $f: X \rightarrow X$ is called strongly transitive if for every non-empty open set $J$ of $X$, there exists an $n$ such that $\bigcup_{k=0}^{n} f^{k}(J)=X$.

We first recall a useful proposition which shows a backward structure of a strongly transitive map for each point; we omit the straightforward proof.

Proposition 3.1. The following conditions are equivalent for a map $f: X \rightarrow X:$

(1) For each $x \in X, \mathrm{Cl} \bigcup_{n=0}^{\infty} f^{-n}(x)=X$.

(2) For every non-empty open subset $U$ of $X, \bigcup_{n=0}^{\infty} f^{n}(U)=X$.

Furthermore, if $f$ is open, then (1) and (2) are equivalent to

(3) If $E \subset X$ is a closed set with $f^{-1}(E) \subseteq E$, then $E=\emptyset$ or $X$.

The examples below clarify the difference between transitivity and strong transitivity.

EXAMPLE 1. There exists a transitive map of the interval which is not strongly transitive. This example appears in [3, Example 3] to illustrate another property. For completeness, we give a construction of the map here.

Let $\left\{p_{n} \mid n \in \mathbb{Z}\right\}$ be a two-sided sequence of real numbers in $[0,1]$ such that

$$
\cdots<p_{-2}<p_{-1}<p_{0}<p_{1}<p_{2}<\cdots,
$$

and $p_{n} \rightarrow 1$ and $p_{-n} \rightarrow 0$ as $n \rightarrow \infty$. For $n \in \mathbb{Z}$ put $I_{n}=\left[p_{n}, p_{n+1}\right]$. Define the $\operatorname{map} f_{n}: I_{n} \rightarrow I_{n-1} \cup I_{n} \cup I_{n+1}$ by

$$
\begin{aligned}
f_{n}\left(p_{n}\right) & =p_{n}, & f_{n}\left(p_{n+1}\right) & =p_{n+1}, \\
f_{n}\left(\frac{2 p_{n}+p_{n+1}}{3}\right) & =p_{n+2}, & f_{n}\left(\frac{p_{n}+2 p_{n+1}}{3}\right) & =p_{n-1},
\end{aligned}
$$

and $f_{n}$ is linear on the intervals complementary to these points. Then $f$ : $[0,1] \rightarrow[0,1]$ is given by $f(0)=0, f(1)=1$, and $f(x)=f_{n}(x)$ if $x \in I_{n}$ (see Figure 2 in [3]). This map is not strongly transitive, because $f^{-1}(0)=0$ (recall Proposition 3.1).

By Example 1 taken mod 1, we also have

EXAMPLE 2. There exists a transitive map of the circle which is not strongly transitive.

Let $B_{n}$ be the bouquet with $n$ petals generated by $n$ copies of the unit circle, where $n \geq 1$. Using Example 1 taken mod 1 and a rotation among petals with respect to the origin, we can easily have an example on $B_{n}$. 
EXAMPLE 3. There exists a transitive map of $B_{n}$ which is not strongly transitive.

EXAMPLE 4 . Since the map $f$ in Example 1 is actually totally transitive as stated in [3, Example 3], we have a totally transitive interval map which is not strongly transitive. On the other hand, the interval map $g$ below is strongly transitive, but not totally transitive:

$$
g(x)= \begin{cases}2 x+1 / 2 & (0 \leq x \leq 1 / 4) \\ -2 x+3 / 2 & (1 / 4 \leq x \leq 3 / 4) \\ 2 x-3 / 2 & (3 / 4 \leq x \leq 1)\end{cases}
$$

(We note that if $I_{1}=[0,1 / 2]$ and $I_{2}=[1 / 2,1]$, then $g\left(I_{1}\right)=I_{2}, g\left(I_{2}\right)=I_{1}$ and $\left.g^{2}\right|_{I_{j}}: I_{j} \rightarrow I_{j}$ is strongly transitive for $j=1,2$.)

4. Main results. Here is our main result.

Theorem 4.1. Let $f: G \rightarrow G$ be a graph map with \# $\operatorname{Fix}\left(f^{k}\right)<\infty$ for each $k \geq 1$. If $f$ is transitive, then it is strongly transitive.

A map $f$ on a graph $G$ is piecewise monotone if there is a finite set $A$ in $G$ such that $f$ is monotone on each component of $G \backslash A$. We note that the $k$ th iterate of a piecewise monotone transitive graph map has at most finitely many fixed points for each $k \geq 1$.

Corollary 4.2. Let $f: G \rightarrow G$ be a piecewise monotone graph map. If $f$ is transitive, then it is strongly transitive.

REMARK. The interval case of the corollary above was proved by CovenMulvey [6].

EXAMPLE 5. Let $f:[0,1] \rightarrow[0,1]$ be the map whose graph appears below. Then $f$ is transitive and the set of fixed points of $f^{k}$ is finite for each $k \geq 1$. Therefore $f$ is strongly transitive, in fact, for each non-degenerate subinterval $J$ of $[0,1]$, there exists an $n$ such that $f^{n}(J)=[0,1]$.

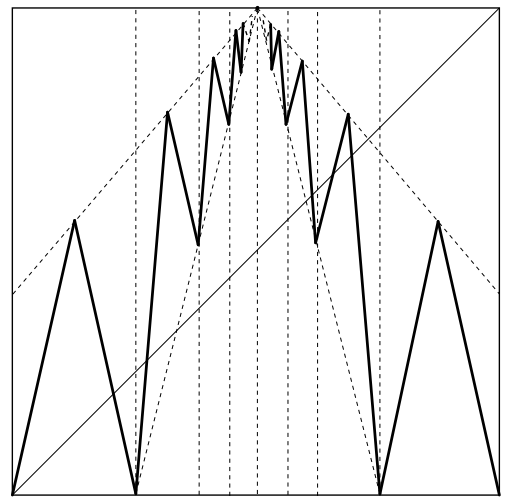


Proof of Theorem 4.1. If $\operatorname{Per}(f)=\emptyset$, then by Theorem 2.1, $f$ is conjugate to an irrational rotation over the circle. Hence $f$ is open and $\bigcup_{n=0}^{\infty} f^{n}(U)=G$ for any non-empty open subset $U$ of $G$. It follows from compactness of $G$ that $f$ is strongly transitive.

We now assume that $\operatorname{Per}(f) \neq \emptyset$; then $\operatorname{Cl} \operatorname{Per}(f)=G$ by Theorem 2.2. In view of Theorem 2.3, we begin by showing that $f$ is strongly transitive when it is totally transitive.

Let $J$ be a connected subset of $G$ with $\operatorname{Int}_{G} J \neq \emptyset$. We have to see that $\left\{f^{n}(J): n \geq 0\right\}$ contains a finite subcovering of $G$.

If $G=\bigcup_{n=0}^{\infty} \operatorname{Int}_{G} f^{n}(J)$, then by compactness of $G$, we have

$$
G=\operatorname{Int} f^{n_{1}}(J) \cup \cdots \cup \operatorname{Int} f^{n_{m}}(J)=f^{n_{1}}(J) \cup \cdots \cup f^{n_{m}}(J)
$$

for some $n_{1}, \ldots, n_{m} \in \mathbb{N} \cup\{0\}$ : thus the conclusion holds in this case.

We next assume that

$$
G \neq \bigcup_{n=0}^{\infty} \operatorname{Int}_{G} f^{n}(J)
$$

Putting $S=G \backslash \bigcup_{n=0}^{\infty} \operatorname{Int}_{G} f^{n}(J)$, we now show that $G \backslash S=\bigcup_{n=0}^{\infty} \operatorname{Int}_{G} f^{n}(J)$ is connected and the set $S$ is finite. Take a periodic point $z \in \operatorname{Int} J$ of period $p$ (Theorem 2.2). Then $\bigcup_{l=0}^{\infty} \operatorname{Int} f^{p l}(J)$ is connected, because Int $J \cap$ Int $f^{p l}(J) \neq \emptyset$ for every $l \geq 1$. (If $z \in \operatorname{Int} f^{p l}(J)$, then this is trivial; if $z \notin \operatorname{Int} f^{p l}(J)$, then $z \in \operatorname{Bd} f^{p l}(J)=\operatorname{Bd} \operatorname{Int} f^{p l}(J)$, thus the intersection is non-empty.) We now find that

$$
\bigcup_{l=0}^{\infty} \operatorname{Int} f^{p l}(J) \subseteq \bigcup_{n=0}^{\infty} \operatorname{Int} f^{n}(J)=G \backslash S \subseteq G=\mathrm{Cl} \bigcup_{l=0}^{\infty} \operatorname{Int} f^{p l}(J) .
$$

Here, the last equality follows from transitivity of $f^{p}$ (by total transitivity of $f$ ) and the Remark following the definition of transitivity in Section 2. Therefore, $G \backslash S$ is connected. Since $S$ contains no non-degenerate intervals by transitivity of $f$, it must be finite.

Our task is now to show that

(1) for every $x \in S$, there exist an open neighborhood $K_{x}$ of $x$ in $G$ and $n_{x} \in \mathbb{N} \cup\{0\}$ such that $x \in K_{x} \subseteq f^{0}(J) \cup \cdots \cup f^{n_{x}}(J)$.

Let $x \in S$. When there is an $l \geq 1$ such that $f^{-l}(x) \cap S=\emptyset$, then by closedness of $f^{l}$, we have an open neighborhood $K$ of $x$ in $G$ such that

$$
f^{-l}(x) \subseteq f^{-l}(K) \subseteq f^{-l}(\mathrm{Cl} K) \subseteq G \backslash S .
$$

By compactness there exists an $m_{x} \geq 0$ satisfying

$$
f^{-l}(\mathrm{Cl} K) \subseteq f^{0}(J) \cup \cdots \cup f^{m_{x}}(J),
$$

hence,

$$
x \in K \subseteq \mathrm{Cl} K \subseteq f^{l+0}(J) \cup \cdots \cup f^{l+m_{x}}(J) .
$$


Next, we assume that $f^{-l}(x) \cap S \neq \emptyset$ for every $l \geq 1$; this is the hardest case. Since $\# S<\infty$ we can take a natural number $k$ such that $f^{k}(x)=x$. We choose a small tree neighborhood $T$ of $x$ in $G$ such that

$$
T \cap(\mathrm{B}(G) \cup S)=\{x\},
$$

and represent $T$ by $\operatorname{arcs}\left[x, x_{i}\right]$ with endpoints $x$ and $x_{i}, i=1, \ldots, p$, as

$$
T=\left[x, x_{1}\right] \cup \cdots \cup\left[x, x_{p}\right],
$$

where $\left[x, x_{i}\right] \cap\left[x, x_{j}\right]=\{x\}$ for $i \neq j$. Further, we may assume by continuity of $f$ that for $i \in\{1, \ldots, p\}$,

$$
f^{k i}(T) \text { is a tree and } f^{k i}(T) \cap(\mathrm{B}(G) \cup S)=\{x\} .
$$

We put

$$
P=\left\{\begin{array}{l|l}
i \in\{1, \ldots, p\} & \begin{array}{l}
\text { there exist } j \neq i, n_{i} \in \mathbb{N} \cup\{0\} \text { and } \varepsilon_{i}>0 \\
\text { such that }\left(\left[x, x_{i}\right] \cup\left[x, x_{j}\right]\right) \cap \mathbb{B}\left(x ; \varepsilon_{i}\right) \subseteq f^{n_{i}}(J)
\end{array}
\end{array}\right\} .
$$

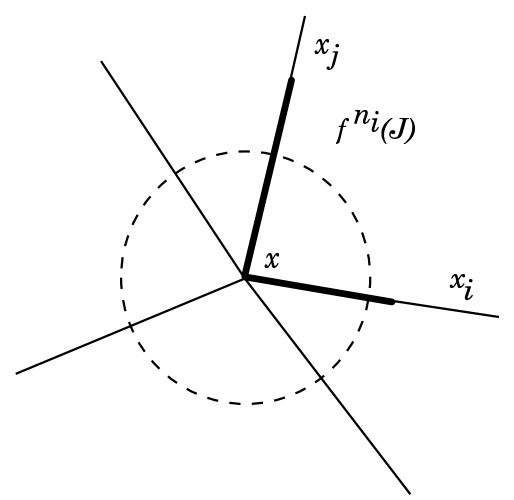

We shall show that for $i \in\{1, \ldots, p\} \backslash P$,

$(4)_{i} \quad$ there exist a non-negative integer $n_{i}$ and an $\varepsilon_{i}>0$ for which

$$
\left[x, x_{i}\right] \cap \mathbb{B}\left(x ; \varepsilon_{i}\right) \subseteq f^{n_{i}}(J) .
$$

Let $i \in\{1, \ldots, p\} \backslash P$. We first prove $(4)_{i}$ under the condition that there is a $\delta>0$ such that

$$
f^{k}\left(\left[x, x_{j}\right]\right) \cap\left(x, x_{i}\right] \cap \mathbb{B}(x ; \delta)=\emptyset \quad \text { for any } j \neq i .
$$

As \# $\operatorname{Fix}\left(f^{k}\right)<\infty$, there is a small tree neighborhood $U$ of $x$ in $T \cap$ $\mathbb{B}(x ; \delta) \subseteq G$ such that

$$
U \cap \operatorname{Fix}\left(f^{k}\right)=\{x\} \quad \text { and } \quad f^{k}(U) \subseteq T .
$$

Then for each $z \in(U \backslash\{x\}) \cap\left[x, x_{i}\right]$, we have

$$
f^{k}(z) \in G \backslash(x, z] .
$$


Indeed, suppose, on the contrary, that there exists a $z_{0} \in(U \backslash\{x\}) \cap\left[x, x_{i}\right]$ such that $f^{k}\left(z_{0}\right) \in\left(x, z_{0}\right]$. We will show that $f^{k}\left(\left[x, z_{0}\right]\right) \subseteq\left[x, z_{0}\right]$, which contradicts the transitivity of $f^{k}$. Indeed, if not, there exists a $y \in\left(x, z_{0}\right)$ with $f^{k}(y) \notin\left[x, z_{0}\right]$. Then by the inclusion in (6), we have either $f^{k}(y) \in\left(z_{0}, x_{i}\right]$ or $f^{k}(y) \in\left(x, x_{j}\right]$ for some $j \neq i$. However, neither can occur by the following reasoning. If $f^{k}(y) \in\left(z_{0}, x_{i}\right]$, then there exists a $y^{\prime} \in\left(y, z_{0}\right) \subseteq U$ such that $f^{k}\left(y^{\prime}\right)=y^{\prime}$, contrary to the first statement of $(6)$. If $f^{k}(y) \in\left(x, x_{j}\right]$ for some $j \neq i$, then there exists a $y_{0} \in\left(y, z_{0}\right)$ such that for any $\varepsilon>0$ there exists a $\delta_{\varepsilon}>0$ satisfying $\left(\left[x, x_{i}\right] \cup\left[x, x_{j}\right]\right) \cap \mathbb{B}\left(x ; \delta_{\varepsilon}\right) \subseteq f^{k}\left(\left[x, x_{i}\right] \cap \mathbb{B}\left(y_{0} ; \varepsilon\right)\right)$ and $f^{k}\left(y_{0}\right)=x$. Take an $n_{0}$ and an $\varepsilon>0$ such that $\left[x, x_{i}\right] \cap \mathbb{B}\left(y_{0} ; \varepsilon\right) \subseteq f^{n_{0}}(J)$ (note $\left.y_{0} \notin S\right)$. Then $\left(\left[x, x_{i}\right] \cup\left[x, x_{j}\right]\right) \cap \mathbb{B}\left(x ; \delta_{\varepsilon}\right) \subseteq f^{k+n_{0}}(J)$. This contradicts the assumption that $i \notin P$.

Moreover, for each $z \in(U \backslash\{x\}) \cap\left[x, x_{i}\right]$ we have

$$
(x, z] \cap f^{k}(G \backslash U) \neq \emptyset .
$$

Indeed, suppose not; that is, assume that there exists a $z_{0} \in(U \backslash\{x\}) \cap$ $\left[x, x_{i}\right]$ such that $\left(x, z_{0}\right] \cap f^{k}(G \backslash U)=\emptyset$. Then we have the conclusion $f^{-k}\left(\left(x, z_{0}\right]\right) \subseteq\left(x, z_{0}\right]$, contradicting the transitivity of $f^{k}$; indeed, if there is a $z_{1} \in f^{-k}\left(\left(x, z_{0}\right]\right) \backslash\left(x, z_{0}\right]$, then $f^{k}\left(z_{1}\right) \in\left(x, z_{0}\right] \subseteq U \subseteq T \cap \mathbb{B}(x ; \delta)$. This implies $z_{1} \in U \backslash\{x\}$. Then if $z_{1} \in\left[x, x_{i}\right]$, we have $f^{k}\left(z_{1}\right) \in G \backslash\left(x, z_{1}\right] \subseteq G \backslash$ $\left(x, z_{0}\right]$ by $(7)$; if $z_{1} \in\left[x, x_{j}\right]$ for some $j \neq i$, we have $f^{k}\left(z_{1}\right) \notin\left(x, x_{i}\right] \cap \mathbb{B}(x ; \delta)$ by $(5)_{i}$. In any case, it is a contradiction.

By (8), we can choose $y_{n}, y \in G \backslash U, n \in \mathbb{N}$, such that

(i) $\lim _{n \rightarrow \infty} y_{n}=y$,

(ii) $\#\left\{f^{k}\left(y_{n}\right) \mid n \in \mathbb{N}\right\}=\infty$,

(iii) $\lim _{n \rightarrow \infty} f^{k}\left(y_{n}\right)=f^{k}(y)=x$ on $\left[x, x_{i}\right]$.

If $y \notin S$, then there exist numbers $n$ and $m_{0}$ such that $y_{m}, y \in f^{n}(J)$ for any $m \geq m_{0}$. Thus, we have an $\varepsilon_{i}>0$ such that $\left[x, x_{i}\right] \cap \mathbb{B}\left(x ; \varepsilon_{i}\right) \subseteq f^{k}\left(f^{n}(J)\right)$.

If $y \in S$, then it follows from $f^{k}(x)=x, f^{k}(y)=x$ and $y \neq x$ that there is an $l \geq 1$ such that $f^{-l}(y) \cap S=\emptyset$. From the same argument as in the proof above, $y$ has an open neighborhood which is covered by finitely many $f^{m}(J)$ 's, so there exist numbers $n$ and $n_{1}<n_{2}<\cdots$ such that $y_{n_{j}}, y \in$ $f^{n}(J)$. Thus, we again have an $\varepsilon_{i}>0$ such that $\left[x, x_{i}\right] \cap \mathbb{B}\left(x ; \varepsilon_{i}\right) \subseteq f^{k}\left(f^{n}(J)\right)$.

We must next examine the statement $(4)_{i}$ in the case when $(5)_{i}$ is not satisfied; that is, for each $\delta>0$, there exists a $j \neq i$ such that

$$
f^{k}\left(\left[x, x_{j}\right]\right) \cap\left(x, x_{i}\right] \cap \mathbb{B}(x ; \delta) \neq \emptyset .
$$

Then we note by the first assumption in (3) that there exists an $i_{1} \neq i$ such that

$$
\left[x, x_{i}\right] \cap \mathbb{B}\left(x ; \delta_{i_{1}}\right) \subseteq f^{k}\left(\left[x, x_{i_{1}}\right]\right)
$$

for some $\delta_{i_{1}}>0$. 
Whenever $\left[x, x_{i}\right] \cap \mathbb{B}\left(x ; \delta_{i_{1}}\right) \subseteq f^{k}\left(\left(x, x_{i_{1}}\right]\right)$, there is a $y \in\left(x, x_{i_{1}}\right]$ such that

(i) $f^{k}(y)=x$,

(ii) for each $\gamma>0$, there exists an $\eta(\gamma)>0$ such that $\left[x, x_{i}\right] \cap \mathbb{B}(x ; \eta(\gamma))$ $\subseteq f^{k}\left(\left[x, x_{i_{1}}\right] \cap \mathbb{B}(y ; \gamma)\right)$.

Since $y \in G \backslash S$ by (2), there is an $n$ such that $y \in \operatorname{Int} f^{n}(J)$. Thus, we have a $\gamma>0$ such that $\mathbb{B}(y ; \gamma) \subseteq f^{n}(J)$. Therefore $\left[x, x_{i}\right] \cap \mathbb{B}(x ; \eta(\gamma)) \subseteq f^{k}\left(f^{n}(J)\right)$.

Next, if $\left[x, x_{i}\right] \cap \mathbb{B}\left(x ; \delta_{i_{1}}\right) \not f^{k}\left(\left(x, x_{i_{1}}\right]\right)$, then $x \notin f^{k}\left(\left(x, x_{i_{1}}\right]\right)$ by (9). Thus, we have a $\gamma_{i_{1}}>0$ such that

$$
f^{k}\left(\left[x, x_{i_{1}}\right] \cap \mathbb{B}\left(x ; \gamma_{i_{1}}\right)\right) \subseteq\left[x, x_{i}\right] .
$$

We note by transitivity of $f^{k}$ that for any $\left(\gamma_{i_{1}} \geq\right) \eta>0$, there exists a $\xi_{i_{1}}(\eta)>0$ such that

$$
\left[x, x_{i}\right] \cap \mathbb{B}\left(x ; \xi_{i_{1}}(\eta)\right) \subseteq f^{k}\left(\left[x, x_{i_{1}}\right] \cap \mathbb{B}(x ; \eta)\right) .
$$

If $i_{1} \in P$, then there exist an $n_{i_{1}} \in \mathbb{N} \cup\{0\}$ and an $\varepsilon_{i_{1}}>0$ such that $\left[x, x_{i_{1}}\right] \cap \mathbb{B}\left(x ; \varepsilon_{i_{1}}\right) \subseteq f^{n_{i_{1}}}(J)$. Thus, we have

$$
\left[x, x_{i}\right] \cap \mathbb{B}(x ; \xi(\eta)) \subseteq f^{k}\left(\left[x, x_{i_{1}}\right] \cap \mathbb{B}(x ; \eta)\right) \subseteq f^{k}\left(f^{n_{i_{1}}}(J)\right),
$$

where $\eta=\min \left\{\varepsilon_{i_{1}}, \gamma_{i_{1}}\right\}$.

If $i_{1} \notin P$, then we begin proving $(4)_{i_{1}}$ by the same process as above. Note that by $(10)_{i}^{i_{1}}$ statement $(4)_{i_{1}}$ implies $(4)_{i}$.

With this strategy, it will again remain to prove our statement in the following case:

- there exist an $i_{2} \neq i_{1}$ and a $\delta_{i_{2}}>0$ such that $\left[x, x_{i_{1}}\right] \cap \mathbb{B}\left(x ; \delta_{i_{2}}\right) \subseteq$ $f^{k}\left(\left[x, x_{i_{2}}\right]\right)$,

- $\left[x, x_{i_{1}}\right] \cap \mathbb{B}\left(x ; \delta_{i_{2}}\right) \nsubseteq f^{k}\left(\left(x, x_{i_{2}}\right]\right)$,

- there exists a $\gamma_{i_{2}}>0$ such that $f^{k}\left(\left[x, x_{i_{2}}\right] \cap \mathbb{B}\left(x ; \gamma_{i_{2}}\right)\right) \subseteq\left[x, x_{i_{1}}\right]$,

- for any $\left(\gamma_{i_{2}} \geq\right) \eta>0$, there exists a $\xi_{i_{2}}(\eta)>0$ such that

$(10)_{i_{1}}^{i_{2}}$

$$
\left[x, x_{i_{1}}\right] \cap \mathbb{B}\left(x ; \xi_{i_{2}}(\eta)\right) \subseteq f^{k}\left(\left[x, x_{i_{2}}\right] \cap \mathbb{B}(x ; \eta)\right),
$$

- $i_{2} \notin P$.

We note again by $(10)_{i_{1}}^{i_{2}}$ that $(4)_{i_{2}} \Rightarrow(4)_{i_{1}}$. Continuing this process, as $\#\{1, \ldots, p\} \backslash P<\infty$, we must finally examine our statement in the following cyclic case:

- there exist pairwise distinct $i_{0}(\equiv i), i_{1}, \ldots, i_{r-1} \in\{1, \ldots, p\} \backslash P$ and positive numbers $\delta_{i_{0}}, \ldots, \delta_{i_{r-1}}$ such that $\left[x, x_{i_{j}}\right] \cap \mathbb{B}\left(x ; \delta_{i_{j+1}}\right) \subseteq$ $f^{k}\left(\left[x, x_{i_{j+1}}\right]\right)(\bmod r)$,

- $\left[x, x_{i_{j}}\right] \cap \mathbb{B}\left(x ; \delta_{i_{j+1}}\right) \nsubseteq f^{k}\left(\left(x, x_{i_{j+1}}\right]\right)(\bmod r)$,

- there exist positive numbers $\gamma_{i_{0}}, \ldots, \gamma_{i_{r-1}}$ such that $f^{k}\left(\left[x, x_{i_{j+1}}\right] \cap\right.$ $\left.\mathbb{B}\left(x ; \gamma_{i_{j+1}}\right)\right) \subseteq\left[x, x_{i_{j}}\right](\bmod r)$, 
- for any $\eta>0$, there exist positive numbers $\xi_{i_{0}}(\eta), \ldots, \xi_{i_{r-1}}(\eta)$ such that

$(10)_{i_{j}}^{i_{j+1}}$

$$
\left[x, x_{i_{j}}\right] \cap \mathbb{B}\left(x ; \xi_{i_{j+1}}(\eta)\right) \subseteq f^{k}\left(\left[x, x_{i_{j+1}}\right] \cap \mathbb{B}(x ; \eta)\right),
$$

- $(4)_{i_{0}} \Rightarrow(4)_{i_{r-1}} \Rightarrow \cdots \Rightarrow(4)_{i_{1}} \Rightarrow(4)_{i_{0}}$.

Then we have a positive number $\gamma$ such that for any $j \in\{0, \ldots, r-1\}$,

$$
f^{k r}\left(\left[x, x_{i_{j}}\right] \cap \mathbb{B}(x, \gamma)\right) \subseteq\left[x, x_{i_{j}}\right] .
$$

Therefore we are able to finish our proof by showing $(4)_{i_{0}}$ under the condition: there is a $\delta>0$ such that

$$
f^{k r}\left(\left[x, x_{j}\right]\right) \cap\left(x, x_{i_{0}}\right] \cap \mathbb{B}(x ; \delta)=\emptyset \quad \text { for any } j \neq i_{0},
$$

and this follows from the same argument as in the proof of $(4)_{i}$ under $(5)_{i}$. This completes the proof of $(4)_{i}$ for $i \in\{1, \ldots, p\} \backslash P$ and the proof of statement (1).

Since $G \backslash \bigcup_{x \in S} K_{x}$ is compact, there exist $n_{1}, \ldots, n_{m} \in \mathbb{N} \cup\{0\}$ such that $G \backslash \bigcup_{x \in S} K_{x} \subseteq f^{n_{1}}(J) \cup \cdots \cup f^{n_{m}}(J)$. Thus we have

$$
G=\left(f^{0}(J) \cup \cdots \cup f^{n_{S}}(J)\right) \cup\left(f^{n_{1}}(J) \cup \cdots \cup f^{n_{m}}(J)\right),
$$

where $n_{S}=\max \left\{n_{x} \mid x \in S\right\}$. Therefore $f$ is strongly transitive.

We must next show this theorem in the case when $f$ is not totally transitive. This follows easily from the first case and the splitting theorem 2.3, and the proof of Theorem 4.1 is finally finished.

Proposition 4.3. Let $f: T \rightarrow T$ be a totally transitive tree map. Then $f$ is strongly transitive if and only if for every non-degenerate connected subset $J$ of $T$, there exists an $M$ such that $f^{m}(J)=T$ for any $m \geq M$.

Proof. The sufficiency is clear. We show the necessity. Assume that $f$ is strongly transitive. Let $J$ be a non-degenerate connected set of $T$. We denote the set of all end points of $T$ by $\mathrm{E}(T)=\left\{e_{1}, \ldots, e_{k}\right\}$. By Proposition 3.1, for each $i=1, \ldots, k$, there exist an $n_{i}$ and a $z_{i} \in T \backslash \mathrm{E}(T)$ such that $f^{n_{i}}\left(z_{i}\right)=e_{i}$. Let $K$ be the smallest subtree of $T$ containing $\left\{z_{1}, \ldots, z_{k}\right\}$. Since $f$ is totally transitive, it follows from [9, Lemma 6] that there exists an $N$ such that $K \subseteq f^{n}(J)$ for any $n \geq N$. Then we have $f^{n+n_{0}}(J)=T$ for any $n \geq N$, where $n_{0}=\max \left\{n_{1}, \ldots, n_{k}\right\}$, since for any $n \geq N$ and $i \in\{1, \ldots, k\}$,

$$
e_{i}=f^{n_{i}}\left(z_{i}\right) \in f^{n_{i}}(K) \subseteq f^{n_{i}}\left(f^{n+\left(n_{0}-n_{i}\right)}(J)\right)=f^{n+n_{0}}(J) .
$$

The following generalizes the result for interval maps of Coven-Mulvey [6] to one for tree maps.

TheOREM 4.4. Let $f: T \rightarrow T$ be an onto tree map. Let $\mathrm{v}(T)$ be the maximum order of any branch point in $T$ and $N_{\mathrm{v}(T)}$ the least common multiple of $\{2, \ldots, \mathrm{v}(T)\}$. Then the following are equivalent: 
(1) $f$ is transitive and has a point of period which is prime to $2, \ldots, \mathrm{v}(T)$.

(2) $f^{N_{\mathrm{v}(T)}}$ is transitive.

(3) $f$ is totally transitive.

(4) $f$ is topologically mixing.

Furthermore, if \# $\operatorname{Fix}\left(f^{k}\right)$ is finite for each $k \geq 1$, then the above are equivalent to

(5) for every non-degenerate connected subset $J$ of $T$, there exists an $M$ such that $f^{m}(J)=T$ for any $m \geq M$.

Proof. The equivalences $(1) \Leftrightarrow(2) \Leftrightarrow(3) \Leftrightarrow(4)$ are well known [8, Theorem 4.1], [1]. The implication $(3) \Rightarrow(5)$ if $\# \operatorname{Fix}\left(f^{k}\right)$ is finite for each $k \geq 1$ follows from Theorem 4.1 and Proposition 4.3. The converse implication $(5) \Rightarrow(3)$ is trivial.

5. Remarks. (I) It is useful to investigate the relation between the dynamics of a graph map and the dynamics of the induced self-homeomorphism of the inverse limit space [2], [3].

Let $f: X \rightarrow X$ be an onto map. Associated with $f$ is the inverse limit space $(X, f)=\left\{\left(x_{0}, x_{1}, \ldots\right) \mid x_{i} \in X\right.$, and $\left.f\left(x_{i+1}\right)=x_{i}\right\}$, and the induced homeomorphism $\widehat{f}:(X, f) \rightarrow(X, f)$ (called the shift homeomorphism), given by $\widehat{f}\left(\left(x_{0}, x_{1}, \ldots\right)\right)=\left(f\left(x_{0}\right), x_{0}, x_{1}, \ldots\right)$.

Proposition 5.1. Let $f: X \rightarrow X$ be an onto map of a metrizable compact space $X$. If the shift homeomorphism $\widehat{f}:(X, f) \rightarrow(X, f)$ is strongly transitive, then $f$ is strongly transitive.

Proof. Note that $\pi_{0} \circ \widehat{f}=f \circ \pi_{0}$, where $\pi_{0}:(X, f) \rightarrow X$ is the projection to the first coordinate space. Let $U$ be an open subset of $X$. Since $\widehat{f}$ is strongly transitive, there exists an $n \in \mathbb{N}$ such that

$$
(X, f)=\bigcup_{k=0}^{n} \hat{f}^{k}\left(\pi_{0}^{-1}(U)\right)=\bigcup_{k=0}^{n} \pi_{0}^{-1} f^{k}(U)=\pi_{0}^{-1}\left(\bigcup_{k=0}^{n} f^{k}(U)\right) .
$$

Therefore we have $\bigcup_{k=0}^{n} f^{k}(U)=X$.

Unfortunately, the shift homeomorphism of a strongly transitive graph map is not always strongly transitive. In fact, we have the following.

Proposition 5.2. Let $G$ be a non-degenerate graph and $f: G \rightarrow G$ be an onto map. Then the shift homeomorphism $\widehat{f}:(G, f) \rightarrow(G, f)$ is strongly transitive if and only if $G$ is the circle and $f$ is conjugate to an irrational rotation.

Proof. The sufficiency is clear. We show the necessity. Assume that $\widehat{f}$ is strongly transitive. We note that $f$ is (strongly) transitive. By Theorem 2.1, 
it suffices to prove that $f$ has no periodic points. Suppose, on the contrary, that $\operatorname{Per}(f) \neq \emptyset$. Let $x \in \operatorname{Per}(f)$ have period $n$. Then the point $\mathbf{x}=\left(f^{n-1}(x), \ldots, f(x), x, f^{n-1}(x), \ldots, f(x), x, \ldots\right)$ of $(G, f)$ is periodic under $\widehat{f}$. Since strong transitivity of $\widehat{f}$ implies its minimality, this contradicts the fact that $\#(G, f)=\infty$.

(II) We note that statement (2) in Proposition 3.1, which was introduced by Parry [7], implies strong transitivity for tree maps.

Proposition 5.3. Let $f: T \rightarrow T$ be an onto tree map. Then $f$ is strongly transitive if and only if $\bigcup_{n=0}^{\infty} f^{n}(U)=T$ for every non-empty open subset $U$ of $T$.

Proof. The necessity is trivial. We show the sufficiency. Suppose that the condition is satisfied. Let $U$ be any non-empty open connected set in $T$. Take a fixed point $x_{0} \in T$ of $f$. Then, by the assumed condition, $x_{0} \in f^{n_{0}}(U)$ for some $n_{0}$. For the endpoints $\mathrm{E}(T)=\left\{e_{1}, \ldots, e_{k}\right\}$ of the tree $T$, it follows from our condition and Proposition 3.1 that for each $i \in\{1, \ldots, k\}, \mathrm{Cl} \bigcup_{n=0}^{\infty} f^{-n}\left(e_{i}\right)=T$ and Int $f^{-n}\left(e_{i}\right)=\emptyset$. Thus, for each $i \in\{1, \ldots, k\}$, there exists an $n_{i} \geq n_{0}$ such that $f^{-n_{i}}\left(e_{i}\right) \cap U \neq \emptyset$. Therefore, $T=\bigcup_{i=1}^{k}\left[e_{i}, x_{0}\right]=\bigcup_{i=1}^{k} f^{n_{i}}(U)$, where $\left[e_{i}, x_{0}\right]$ denotes the smallest arc with endpoints $e_{i}$ and $x_{0}$ in $T$.

However, this is not always true for a general graph map.

EXAMPLE 6. Let $f:[0,1] \rightarrow[0,1]$ be the map whose graph appears below. Using it, we define the circle map $g: S^{1} \rightarrow S^{1}$ by $g\left(e^{2 \pi i \theta}\right)=e^{2 \pi i f(\theta)}$, where $0 \leq \theta \leq 1$. Then $g$ is transitive and satisfies statement (2) in Proposition 3.1, but is not strongly transitive. (Note that if $J$ is a non-degenerate connected set in $S^{1}$ not containing the point $(1,0)$, then for any $n$, there is no $0<\varepsilon<1$ such that $\left\{e^{2 \pi i \theta} \mid 1-\varepsilon<\theta<1\right\} \subseteq g^{n}(J)$.)

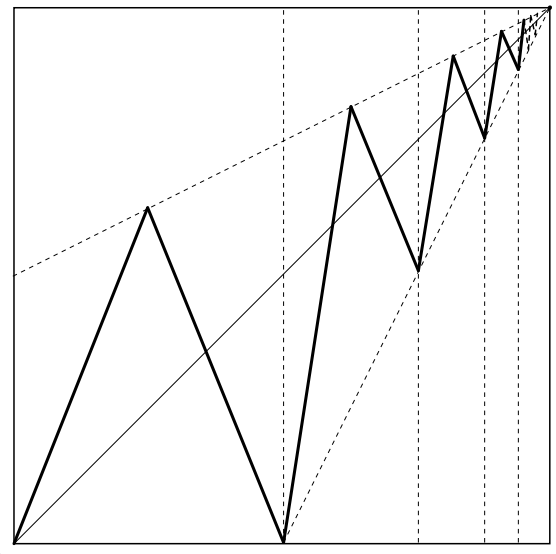




\section{References}

[1] Ll. Alsedà, M. A. del Río, and J. A. Rodríguez, A splitting theorem for transitive maps, J. Math. Anal. Appl. 232 (1999), 359-375.

[2] M. Barge and J. Martin, Chaos, periodicity, and snakelike continua, Trans. Amer. Math. Soc. 289 (1985), 355-365.

[3] -, -, Dense orbits on the interval, Michigan Math. J. 34 (1987), 3-11.

[4] A. M. Blokh, On transitive mappings of one-dimensional branched manifolds, in: Differential-Difference Equations and Problems of Mathematical Physics, Akad. Nauk Ukrain. SSR, Inst. Mat. Kiev, 1984, 3-9, 131 (in Russian).

[5] - The connection between entropy and transitivity for one-dimensional mappings, Uspekhi Mat. Nauk 42 (1987), no. 5, 209-210 (in Russian).

[6] E. M. Coven and I. Mulvey, Transitivity and the centre for maps of the circle, Ergodic Theory Dynam. Systems 6 (1986), 1-8.

[7] W. Parry, Symbolic dynamics and transformations of the unit interval, Trans. Amer. Math. Soc. 122 (1966), 368-378.

[8] R. P. Roe, Dynamics and indecomposable inverse limit spaces of maps on finite graphs, Topology Appl. 50 (1993), 117-128.

[9] —, Dense periodicity on finite trees, Topology Proc. 19 (1994), 237-248.

Katsuya Yokoi

Department of Mathematics

Shimane University

Matsue, 690-8504, Japan

E-mail: yokoi@riko.shimane-u.ac.jp

Received September 29, 2005;

received in final form January 21, 2006 